\title{
El merchandising, las TIC y la publicidad en la gestión de ciudades turísticas.
}

\author{
Merchandising, ICT and advertising from in the management of tourist \\ cities.
}

Ing. Ernesto Cabezas García ${ }^{1}$

\section{Resumen.}

En el presente artículo, se realiza una valoración paradójica sobre el impacto de las reglas y técnicas del merchandising, las Tecnologías de la Información y la Comunicación, y la publicidad en la mejora continua de las ciudades, como vía para incrementar su competitividad y posicionamiento en el mercado turístico, objetivo en el que se enmarca la investigación realizada, a partir del empleo de método teóricos conocidos, cuyos resultados permiten establecer una relación funcional estratégica entre el merchandising, las Tecnologías de la Información y la Comunicación, y la publicidad, en la consolidación de las ciudades turísticas.

Palabras claves: Merchandising, TIC, publicidad, ciudades, turismo.

\begin{abstract}
.
In this article, a paradoxical assessment is made about the impact of the rules and techniques of merchandising, Information and Communication Technologies, and advertising in the continuous improvement of cities, as a way to increase their competitiveness and positioning in the tourism market, objective in which the research carried out is framed, based on the use of known theoretical methods, whose results allow establishing a strategic functional relationship between merchandising, Information and Communication Technologies, and advertising, in the consolidation of tourist cities.
\end{abstract}

Keywords: Merchandising, ICT, advertising, cities, tourism.

\section{Introducción}

Desde tiempos remotos, el intercambio de bienes y servicios, ha jugado un papel determinante en el desarrollo y perduración de la humanidad. Las bases económicas que sustentan las distintas estructuras políticas y sociales del mundo, tuvieron su origen en el

${ }^{1}$ Universidad de la Habana, Facultad de Turismo. La Habana, Cuba, ernesto.cg@ehtpe.tur.cu 
incremento de la oferta y la demanda, motivado por su capacidad para mantener a buen ritmo, la mejora de la calidad de vida y el avance, hacia el logro de una sostenibilidad consciente y comprometida.

En este entorno evolutivo, las ciudades se han convertido en núcleos esenciales de la relación cliente-proveedor, cuya formación económica social (FES), ha contribuido a establecer enfoques y tendencias comerciales, basados en los conocimientos adquiridos de generación en generación.

La satisfacción de un mercado cada vez más creciente, exigente y dispuesto a no tolerar retrocesos, impone con inmediatez, la necesidad de adaptarse a los cambios y actuar en consecuencia para evitar la autodestrucción de los negocios. Bajo esta premisa, emplear estrategias que permitan aumentar la competitividad de las ciudades y los países, se convierte en un elemento de diferenciación que deberá centrar su atención en la preservación de la identidad y el patrimonio como vía para la consolidación de capacidades distintivas.

El vertiginoso desarrollo del turismo, ha revolucionado por completo la visión tradicionalista de las ciudades y sus pobladores, hoy en día las dimensiones del ocio autotélico $^{[2]}$, adquieren una mayor relevancia cuando nuestras urbes son vistas por el turista como una gran feria a recorrer, en busca de experiencias vivenciales gratificantes y productos nativos que adquirir, como recuerdo de su visita. Este panorama, cambia el contexto en el cual se desarrollan las actividades comerciales y su interacción con la sociedad, su modo de vida, sus costumbres y sus características etnológicas.

Todo establecimiento comercial, con independencia de su modalidad o función, posee generalmente varios departamentos y secciones en los que se ubican los productos y/o servicios a ofertar, por ejemplo, una tienda mixta, puede tener un área destinada a implementos de ferretería, otra a equipos electrodomésticos, otra a útiles del hogar, etc., en las cuales el cliente identificará diferencias sensoriales (vista, tacto, gusto, olfato y oído), propias del lugar en que se encuentre, pero desde el punto de vista organizativo, será posible apreciar alguna que otra similitud en cuanto a condiciones de confort, seguridad, iluminación, ventilación, higiene y limpieza, señalética, profesionalidad en la atención de los dependientes, calidad en el surtido, facilidades de pago, protección al consumidor, medios para el reciclaje de desechos y salvaguarda del medio ambiente etc. Esto se debe a la política, estrategia o forma de gestión integrada de la administración del establecimiento, enfocada en garantizar un mínimo de condiciones en todas sus áreas, que permita al cliente una estancia mucho más placentera para facilitar la compra y lograr que éste repita su visita.

Lo anterior, visto desde una perspectiva de ciudad, pudiera resultar un poco difícil de aplicar, pero si visualizamos la metrópolis como una tienda en la que coexisten múltiples departamentos, actividades, productos y servicios, personas, transportes, o cualquier otro componente citadino, podemos establecer una relación paradójica entre las condiciones mínimas que debe garantizar el establecimiento comercial para el bienestar de sus clientes

[2] El ocio autotélico es el ocio puro y desinteresado. La actividad realizada causa satisfacción. "Al dejarse llevar por su interior, se pueden ejercitar opciones, elecciones y decisiones más libres y mantenerse según la naturaleza y necesidades de cada uno" (Cuenca, 2000) 
y como debe funcionar la ciudad en cuanto a los mismos conceptos. Se trata de ver la urbe como una gran oportunidad de venta, en la que cada vivienda, negocio, entidad u organización, será un departamento, sección o área del establecimiento comercial, y como tal, habrá que gestionarla para obtener la mayor rentabilidad, promover sus atributos y beneficios, atraer la mayor cantidad de turistas o clientes y posicionar el destino como una experiencia única e irrepetible.

Hoy en día, el arte de vender y saber comprar, basado en emociones, sensaciones y experiencias, como parte de la evolución de los conceptos relativos a la inteligencia emocional y a los principios psicológicos que intervienen en la actuación del ser humano, determinan en un contexto social dado, la efectividad del marketing mix (producto, precio, plaza, promoción, personas, procesos, presentación y productividad). En el caso específico de los establecimientos comerciales, el merchandising, como parte del marketing dirigida a aumentar el rendimiento económico del punto de venta, constituye el camino a seguir para alcanzar niveles superiores de eficiencia y eficacia en el sector, lo cual, visto desde el enfoque de ciudad, nos permite tener una visión más abarcadora de como incrementar el deseo en los clientes de visitarla, recorrer sus calles, compartir con el pueblo, comprar sus productos y servicios, y generar en su mente experiencias memorables.

Por otra parte, el vertiginoso desarrollo de las Tecnologías de la Información y la Comunicación, comúnmente denominado TIC, es un fenómeno que ha revolucionado completamente la vida del hombre, trayendo consigo cambios de significación en las relaciones laborales, económicas, sociales, culturales, y muy especialmente en la forma de pensar y de actuar de los ciudadanos, por lo que también las ciudades han de transformarse y adaptarse a los nuevos patrones que impone el desarrollo, como vía para avanzar hacia una ciudad más inteligente.

El análisis de los elementos antes expuestos, advierte la necesidad de adoptar nuevas formas de gestión que aseguren la perduración y sostenibilidad de las ciudades. La escasa cultura sobre la aplicación del merchandising, la resistencia al cambio en los locatarios, el miedo al emprendimiento, la falta de financiamiento, la no preservación del patrimonio, el desaprovechamiento de las TIC y el no comprometimiento de las autoridades públicas en la creación de estrategias integrales de desarrollo, son problemáticas que afectan hoy más que nunca, el atractivo de las urbes y su consolidación como destino turístico competitivo. (Gómez, 2014) refiere que la Organización Mundial del Turismo (OMT), concibe el producto turístico como el resultado de su entorno, es decir, que lo que rodea el lugar, configura la imagen que el visitante recordará y llevará en su memoria. Por su parte (Dupeyras y Maccallum, 2015), ratifican que la competitividad de un destino turístico es la capacidad para optimizar su atractivo para residentes y no resientes y proveer servicios turísticos de calidad, innovadores y atractivos. Es por ello que integrar de forma armónica, dinámica y sinérgica la visualidad de las ciudades constituye un factor calve para el éxito de su atractivo, y en ello, el merchandising, puede desempeñar un papel fundamental.

La consulta bibliográfica realizada por el autor, evidencia la novedad del tema que se propone en la presente investigación, al no existir referencias puntuales sobre el aprovechamiento de las reglas y técnicas del merchandising en la gestión integral de las 
ciudades, ya que ello se ha aplicado especialmente a los establecimientos comerciales que en ella coexisten, cada uno enfocado en sus propios intereses y no en los de la urbe de forma general. De ahí que se persiga como objetivo: realizar un análisis teórico sobre el aprovechamiento de las reglas y técnicas del merchandising, las TIC y la publicidad en la gestión de las ciudades turísticas, como vía para incrementar su atractivo, competitividad y posicionamiento. El estudio realizado se encuentra sustentado en la aplicación de método teóricos conocidos, cuyos resultados permiten establecer una relación funcional estratégica entre el merchandising, las Tecnologías de la Información y la Comunicación, y la publicidad, en la consolidación de las metrópolis turísticas.

\section{Métodos}

Desde una concepción dialéctico-materialista, la investigación realizada se apoya fundamentalmente en la aplicación de métodos teóricos. El autor, aprovecha los argumentos ofrecidos por (Valcárcel et al, 2015) y se nutre de las experiencias de (Rivero, 2011), (Martínez, 2015), (Trujillo, 2015) y (Cabrera, 2017) en la ejecución de los siguientes métodos:

-Analítico-sintético: se utilizó para la conformación del marco teórico-conceptual sobre el aprovechamiento de las reglas y técnicas del merchandising, las TIC y la publicidad en la gestión de las ciudades turísticas.

-Histórico-lógico: se empleó en el análisis evolutivo del merchandising, las ciudades, las TIC y su papel en la formación de la identidad visual y configuración del entorno. -Sistémico estructural funcional: permitió establecer las relaciones dialécticas entre el merchandising, las TIC y la publicidad, y su vínculo con los problemas que afectan hoy en día el atractivo de las ciudades.

-Hipotético-deductivo: facilitó la identificación de la relación funcional entre las reglas y técnicas del merchandising, las TIC y la publicidad, en la gestión de las ciudades turísticas, como vía para incrementar su atractivo, competitividad y posicionamiento.

-Modelación: viabilizó la abstracción del autor en la representación hipotética de la relación funcional entre las reglas y técnicas del merchandising, las TIC y la publicidad, en la gestión de las ciudades turísticas.

\section{Resultados}

Se presenta un análisis teórico conceptual sobre el aprovechamiento de las reglas y técnicas del merchandising, las TIC y la publicidad en la gestión de las ciudades turísticas, cuyo estudio evidenció la existencia de una relación funcional estratégica entre estos elementos, como vía para incrementar el atractivo, competitividad y posicionamiento de las metrópolis, lo cual se detalla en la discusión del presente artículo.

\section{Discusión}

De acuerdo con la bibliografía consultada, el término merchandising no posee traducción exacta en español, pues aunque generalmente se identifica como micro-mercadotecnia, es un préstamo del inglés, compuesto por la palabra marketing y la terminación -ing, que indica la acción voluntaria para conseguir un objetivo). Merchandising, procede a su vez 
del verbo merchandise (comercializar o mercancía en determinados contextos), tomado del francés antiguo y derivado de marchand (vendedor o comerciante).

Según la definición del Instituto Francés de Merchandising, es el conjunto de estudios y de técnicas de aplicación y puesta en práctica, separada o conjuntamente, por los distribuidores y los productores con el fin de incrementar la rentabilidad del punto de venta y la circulación de productos a través de una adaptación permanente del surtido a las necesidades del mercado, y a través de la presentación apropiada a los productos. (González, 2015)

En tanto, el Dr. Charles Kepner fundador de la Compañía Kepner-Tregoe dedicada a la consultoría de gestión, plantea que el merchandising es la mercancía que conviene, en el lugar que conviene, en la época que conviene, en las cantidades convenientes y al precio que conviene. (González, 2015)

Como se ha visto anteriormente, el objetivo fundamental del merchandising se orienta al incremento de la rentabilidad del punto de venta, lo cual, a partir de la bibliografía estudiada se puede desagregar en varias acciones: llamar la atención, informar y dirigir al cliente hacia el producto, incentivar la compra por impulso y garantizar un mayor nivel de ventas, propiciar una mayor rotación de los productos y una adecuada gestión de los surtidos, potenciar la venta de los productos estrella, imán e impulso ${ }^{[3]}$, lograr la venta de stocks o productos antes no visibles, y aprovechar las potencialidades del punto de venta en todos los aspectos, para lograr una mayor atracción sensorial del cliente. Siguiendo los criterios de (Mouton, 1989), (Salen, 1994) y (León, 2015), se puede afirmar que la importancia del merchandising está dada por su capacidad para optimizar el manejo de los productos y determinar las ubicaciones adecuadas en función de variables como $\boldsymbol{e l}$ lugar, el tiempo, la forma, la cantidad y la arquitectura interior y exterior del establecimiento. La mayoría de los autores, reconocen la existencia de tres tipos básicos de merchandising, en los cuales se enmarcan el grosso de sus reglas y técnicas. Siguiendo los postulados de (Salen, 1994), (Intalentia Innovación, 2013) los describe como sigue:

- Merchandising visual o de presentación: es la manera de presentar los artículos y la tienda de modo que el acto de compra sea lo más fácil para el cliente y lo más rentable posible para el comerciante, todo lo que se ve, se vende; todo lo que se coge, se compra.

- Merchandising de gestión: se gestiona el espacio para obtener el máximo rendimiento posible del lineal (espacio destinado por el establecimiento a la exposición y venta de los productos), determinando su tamaño, las distintas familias, marcas y artículos que los forman, teniendo en cuenta la rotación de los productos, el rendimiento por metro cuadrado de la superficie de venta, el rendimiento por metro de lineal, etc.

\footnotetext{
[3] Productos estrella: aquellos por el que se caracteriza especialmente el establecimiento, el punto fuerte de la tienda. Productos imán: aquellos que atraen la atención o despiertan la curiosidad del cliente, pero que no son necesariamente los más vendidos. Productos impulso: productos generalmente de bajo coste que se compran fácilmente de forma no planificada. (Luksdesignshop, 2013)
} 
- Merchandising de seducción: basado en la denominada «tienda espectáculo», tiene en cuenta la concepción del mobiliario específico, la decoración, la información, etc., con el objetivo de dar un aspecto seductor al lineal y a la tienda, para promover la imagen del propio distribuidor, frecuentemente se realizan animaciones en el punto de venta en las que el cliente interactúa con los productos.

De acuerdo con el famoso escritor, filósofo y vlogger ${ }^{[4]}$ suizo Alain de Botton, fundador de la School of Life y de la Living Architecture, existen seis puntos o cualidades a tener en cuenta para hacer una ciudad atractiva, los cuales se sintetizan a partir de (Rosenfield, 2015) y (SalvoLomas, 2015) de la siguiente forma:

1-Variedad y orden: "El orden significa balance, simetría y repetición. El orden es una de las razones por las que mucha gente ama París. Sin embargo, el orden excesivo puede ser también un problema" (los edificios deben ser uniformes en apariencia y disposición hasta cierto grado).

2-Vida visible: "Hay calles que están muertas y calles que están vivas, por lo general se prefieren aquellas vivas" (es agradable ver a la gente pasear por las calles y trabajar en los escaparates).

3-Compacto: "Las más bellas ciudades compactas tienen plazas. La plaza ideal debe ofrecer un sentido de contenimiento, no claustrofobia" (no expansión dispersa).

4-Orientación y misterio: "Por definición, las ciudades son enormes, pero aquellas que mucha gente ama también tienen pequeñas callejuelas donde el visitante se puede sentir acogido y un tanto perdido" (un balance entre grandes calles y callejuelas debe generar eficiencia en los viajes... y también el perderse en ocasiones);

5-Escala: "Las ciudades modernas siempre son grandes, pero es necesario cuidar la altura de sus construcciones para no romper la belleza del paisaje" (un edificio debe ser de cinco pisos como máximo, a menos que su significancia sea digna de un mayor espacio aéreo).

6-Hazlo local (Make it local): "No se quieren edificios que luzcan igual en todas partes" (Melbourne, por ejemplo debería verse de alguna manera diferente de Barcelona, ya que sus cualidades culturales y geográficas son diferentes).

Al analizar el objetivo del merchandising desde una visión más interiorizada, conjuntamente con los planteamientos de Botton, encontramos una oportunidad estupenda para establecer nuevas formas de hacer que permitan transformar la visualidad

\footnotetext{
[4] Un vlog es un blog que publica sus contenidos en formato vídeo. Se escribe con $V$ por la fusión entre Vídeo+Blog: VLOG. Al responsable máximo de un vlog se le conoce como vlogger. En un vlog, los vídeos se ordenan cronológicamente, igual que los artículos de un blog con contenido en texto normal. Las temáticas de los vlogs son muy variadas, desde recetas y peinados hasta videojuegos y tutoriales. (Blaya, 2018)
} 
de las ciudades y los países. (Morales, 2014) explica que para vender sus productos, el fabricante hace marketing, es decir actúa sobre el consumidor final, mientras que el establecimiento comercial para vender sus productos hace merchandising, actúa sobre el comprador. El merchandising es el marketing del vendedor comercial, es el marketing en el punto de venta. No obstante, debe tenerse en cuenta que el fabricante puede realizar merchandising para lograr una presencia adecuada de sus productos en el punto de venta, lo cual estará en correspondencia, con el embalaje, el diseño del producto, etc.

Lo mismo ocurre, cuando un país trata de mostrar al mundo los atractivos y encantos de su patrimonio nacional, su desarrollo económico y competitividad, o simplemente: ¿cómo vive su pueblo?, ¿qué es lo que les hace especiales, diferentes e interesantes? Ello puede originar en el público, un deseo insaciable de viajar al país para vivir la experiencia que idealizaron en su mente, y es entonces cuando la ciudad que los recibe, logra por sí sola, enamorarlos o perderlos para siempre por no alcanzar sus expectativas. Una vez que el turista arriba a la ciudad, ya no habrá forma de evitar el impacto de ésta sobre sus recuerdos y en tanto, cada espacio de ella, contribuirá a formar una imagen más positiva o negativa del país, y se debe decir país, porque no hablará solo de La Habana, hablará también de Cuba. Las metrópolis no son solo un asentamiento poblacional, constituyen una parte imprescindible de la vida, en la que nace, crece, se desarrolla y puede acabar, una civilización entera. Es por ello que cada una tiene su identidad, estilo o sello personal, y precisamente, aprovechando esa capacidad diferenciadora habrá que trabajar para encontrar nuevas pautas de gestión que permitan alcanzar un desarrollo sostenible perdurable, tarea en la que el merchandising, puede contribuir decisivamente.

Por otra parte, el acelerado crecimiento y generalización de las TIC, impone cambios radicales en la sociedad y en las estructuras y funciones de los componentes citadinos, transformando por completo la vida en las urbes, ya que como explica (Medina, 2016), son el conjunto de procesos y productos derivados de las nuevas herramientas (hardware y software), soportes de la información y canales de comunicación relacionados con el almacenamiento, procesamiento y transmisión en forma digital de la información. Ello implica entonces, un cambio en la forma de gestionar y percibir la organización, ya que el aprovechamiento de las TIC, permite llegar a un mayor número de personas, en el menor tiempo posible y facilitar el acto de compra, conjuntamente asociado a un proceso de retroalimentación más dinámico y efectivo, que permitirá corregir oportunamente las deficiencias existentes, como parte de la mejora continua y evolución propia de los negocios.

El merchandising, ha encontrado en las TIC otra forma de comunicarse con el cliente y transmitir las sensaciones de vida de los comercios, adentrándose en su mundo, obteniendo información cada vez más valiosa para fidelizarlo y en especial cambiando sus gustos y preferencias respecto a los productos y servicios que se ofertan. Este fenómeno, ha provocado que el cliente se convierta en un vendedor involuntario del propio comerciante, ya que al compartir en las webs sus experiencias sobre el producto o servicio comprado, genera rechazo o aceptación en las personas que consultan dicha información, pues el público ha aprendido, que la verdadera realidad del producto, solo la puede trasmitir aquel que lo ha probado, y su valoración al respecto constituye la mayor 
publicidad y convencimiento para efectuar finalmente la compra. El merchandising 2.0, como se le ha llamado a la gestión de esta actividad mediante el aprovechamiento de internet, está cambiando rápidamente y cada vez, gana más aceptación entre los consumidores, se trata de construir una tienda virtual y presentar de forma adecuada según las reglas del merchandising visual, la información gráfica de lo que se quiere vender y con ello contribuir a la decisión de compra del cliente

Los anterior, también aplica a las ciudades y países; diversas plataformas web, almacenan, comparten y propician el debate de las vivencias de los viajeros sobre cualquier destino en particular, en las cuales se puede encontrar una realidad muy distinta de lo publicitado. Las urbes han sido tomadas por las TIC en todos sus ámbitos y esto transforma cada día la interacción de los ciudadanos con la información y su utilización; no resulta casual, que los gobernantes utilicen las redes sociales para comunicarse con su pueblo, de una forma más directa e inmediata, ello es el resultado de los impactos y tendencias que impone el cambio. Las metrópolis, tienen que avanzar hacia un aprovechamiento masivo y consecuente de las TIC, y desarrollar un despliegue intensivo de las mismas en todas sus áreas, de manera que la población residente y los turistas, las perciban e interactúe con ellas, ya sean cajeros automáticos, facilidades de pago mediante tarjetas de crédito o compras por internet, displays interactivos con información relevante para el visitante en varios idiomas (mapas con servicio GPS, hospitales, museos, aeropuertos, unidades policiales, buró de información especializada, paradas de ómnibus y trenes, etc.), publicidad digital de productos y servicios, señalética uniforme y animada en semáforos y áreas específicas de la urbe, empleo de luminarias inteligentes y proyecciones holográficas, así como rótulos digitales diversos, por solo citar algunos ejemplos. Ello posibilitará poco a poco el avance hacia una ciudad inteligente sin perder la identidad que la caracteriza, respetando el patrimonio, al garantizar una convivencia entre lo novedoso y lo histórico que no ponga en peligro la visualidad, muy especialmente en aquellas urbes patrimoniales.

Según (Bouskela et al, 2016) "una Ciudad Inteligente y sostenible es una ciudad innovadora que utiliza las TIC y otros medios para mejorar la toma de decisiones, la eficiencia de las operaciones, la prestación de los servicios urbanos y su competitividad. Al mismo tiempo, procura satisfacer las necesidades de las generaciones actuales y futuras en relación con los aspectos económicos, sociales y medioambientales. Asimismo, resulta atractiva para los ciudadanos, empresarios y trabajadores, pues genera un espacio más seguro, con mejores servicios y con un ambiente de innovación que incentiva soluciones creativas, genera empleos y reduce las desigualdades. De esa manera, las Ciudades Inteligentes promueven un ciclo virtuoso que produce no solo bienestar económico y social, sino también el uso sostenible de sus recursos con miras a elevar la calidad de vida a largo plazo", por lo que, de una forma u otra, todos los asentamientos poblacionales, tendrán que preparar, organizar y ejecutar la migración de la vida citadina, hacia una ciudad más inteligente, mediante el aprovechamiento de las TIC, lo que ya se aprecia en las denominadas ciudades 2.0, basadas fundamentalmente en el incremento de la interacción de los ciudadanos y la administración; incluso ya se vislumbran intentos de ciudad 3.0, a partir de un aumento acelerado y sin pauta de la informatización de la sociedad. 
Como se ha visto, la publicidad es uno de los aspectos que determina la aceptación de los productos y servicios que se ofrecen, y por supuesto, tienen un impacto en las metrópolis y sus poblaciones; de acuerdo con (Breva y Mut, 2016) "existen espacios urbanos donde la presencia de la publicidad exterior ha ido conformando estéticas particulares que hacen que algunos autores afirmen que la historia de la publicidad está ligada a la de las ciudades. Además, desde la neuroarquitectura, mix entre neurociencias y psicología ambiental, se demuestra que el diseño de los espacios arquitectónicos influye en los estados emocionales y el comportamiento de los individuos", lo cual visto desde la aplicación del merchandising, ilustra la necesidad de trabajar cada vez más en la creación de emociones y recuerdos memorables, que conduzcan a garantizar la repitencia de los clientes, y en el caso de las ciudades, la visita de un mayor número de turistas, como resultado del marketing de boca a boca.

La publicidad, grosso modo, es una forma múltiple de comunicar, que utiliza los medios de difusión en forma pagada, para incrementar el consumo de un producto o servicio, así como lanzar, mejorar o reposicionar en la mente del cliente, una marca o producto dentro del mercado de consumo. El merchandising, se apoya en la publicidad para atraer y reforzar la acción de compra, lo que le permite a su vez, diferenciarse de la competencia y establecer patrones de consumo. Es normal entonces, que las ciudades y países también empleen la publicidad para fines específicos, ya que como se expresó anteriormente, puede conducir a la transformación de la sociedad, lo que visto desde un enfoque de las TIC, desemboca en la publicidad 2.0, de banners y ventanas emergentes en los blogs, sitios web, etc., cuyo objetivo es fortalecer la campaña publicitaria mediante un clic; ya últimamente se habla de la publicidad 3.0, basada en una mayor interacción con las redes sociales, con predominio de técnicas y estrategias de innovación para las marcas.

Para el logro de sus objetivos, el merchandising se nutre de un grupo de reglas y técnicas, que desde un enfoque de ciudad y con el aprovechando las TIC, pudieran ayudar a resolver algunos de los problemas de gestión existentes hoy en día, para ello, primeramente debe comprenderse según (Intalentia Innovación, 2013), que las mismas están orientadas a garantizar la disponibilidad del producto, que éste se encuentre en el lugar adecuado, que haya la cantidad necesaria, al precio correcto, visible y en el momento preciso, ya que: sobre el producto actúa el espacio que se le destina, la identificación, la visibilidad, el precio y las ofertas, sobre el entorno la ambientación, la dinámica, la organización general, etc. y sobre sobre el consumidor, porque todos estos medios le informan, le recuerdan, le refuerzan el mensaje, desvían la compra de productos competitivos y actúan en la decisión de compra. Estas reglas y técnicas básicas, pueden enunciarse a partir de (Intalentia Innovación, 2013) y (Merchandising Perú, 2015), como sigue:

Acceso al establecimiento: la entrada al establecimiento es un punto de transición entre el mundo exterior y el negocio, por lo que resulta muy importante que ésta posea una gran accesibilidad (evitar un excesivo número de escalones, suprimir obstáculos, lograr un diseño atractivo que invite a la entrada, etc.), lo más adecuado, sería utilizar puertas trasparentes y de fácil apertura o automáticas. 
Esta regla, vista desde una perspectiva de ciudad, debe comprenderse como la necesidad de lograr cierta uniformidad en todos los rincones de la urbe, en los que prevalezca la limpieza, el orden común y la no obstaculización de puertas y accesos de cualquier tipo con elementos decorativos, plantas, vallas publicitarias o informativas, equipos o cualquier otro componente que pueda dificultar la visibilidad y el flujo normal de los clientes hacia dentro y fuera de los locales. En especial, deben eliminarse las barreras arquitectónicas y velar por el correcto estado del pavimento, las aceras y rampas por las que circularán los ciudadanos y visitantes.

Escaparates exteriores: las vitrinas exteriores permiten exhibir los artículos más llamativos e interesantes a los clientes que transitan por su frente, además de especificar el tipo de establecimiento del que se trata y calar en los gustos y preferencias de los clientes.

Este aspecto se enfoca en la importancia que adquieren las fachadas de cristal de la ciudad y la necesidad de organizar la visualidad de las mismas, para garantizar la transmisión de un mensaje de limpieza, higiene, buen gusto y a la vez modernidad, sin dejar de considerar la armonía de las decoraciones en el contexto. Se debe evitar en lo posible la exhibición de componentes deteriorados, incompletos o discordantes y en el caso de los establecimientos comerciales, garantizar exhibiciones alegres y atractivas a la vista del cliente.

Rótulo del establecimiento: la identificación del comercio es un elemento fundamental que en muchas ocasiones es infravalorado. Se debe prestar una atención especial a su colocación, de modo que presente una gran visibilidad incluso a largas distancias, su aspecto (forma, color, utilización de luces, rótulos digitales, etc.), su contenido (breve y conciso y que dé idea del negocio al que se refiere), etc.

Desde una visión global, resulta necesario evitar la existencia de rótulos y señaléticas deterioradas, rutinarias, monotemáticas y/o carentes de atractivo y creatividad. Se deben utilizar lumínicos de colores sugerentes y llamativos, emplear rótulos digitales de significancia, que permitan destacar aún más las funciones de los distintos edificios o instalaciones existentes.

Normas generales de exhibición: debe colocarse el máximo del producto, en contacto, con el máximo de clientes, durante el mayor tiempo posible. Por tanto, debe tenerse en cuenta la circulación en el establecimiento (flujo). Se trata de situar el producto en lugares donde aumentan las posibilidades de ser adquirido por el consumidor.

De forma general, se hace necesario evitar el desorden en los distintos comercios y locales coexistentes en la ciudad, con relación a la forma de exhibir sus productos y servicios; se debe garantizar en todo momento un flujo circulatorio adecuado y armónico de los clientes, respetar la complementariedad de las familias y subfamilias de productos y no dejar a la vista del consumidor expositores vacíos, así como respetar las formas básicas de implantación y el nivel de presentación de los productos en lo referente a su altura desde el piso.

Circulación en el interior del establecimiento: en la concepción del espacio de venta, se debe repartir el espacio disponible entre las distintas secciones y decidir su ubicación óptima, de manera que se facilite la circulación de los clientes. Las técnicas de exposición 
permiten manejar la venta visual: lo que se ve y está al alcance de las manos, se vende; y lo que se ve en masa, se vende en cantidad.

Resulta imprescindible velar por una correcta distribución espacial a nivel de ciudad de los servicios, evitar almacenar productos que obstruyan el paso o la adecuada circulación dentro y fuera de los locales y calles circundantes, de manera que disminuya la aglomeración de personas en las áreas de mayor concurrencia, así como respetar las calles como pasillos de aspiración hacia cualquier establecimiento o local, etc.

Zonas calientes y frías: se suele admitir la existencia de dos zonas triangulares claramente diferenciales según las corrientes de circulación de la clientela: la zona caliente de circulación natural y la zona fría que será necesario incentivar a través de puntos calientes, para que los consumidores circulen por ella.

En las metrópolis, resulta prácticamente imposible evitar la existencia de zonas poco y/o altamente transitadas, se debe buscar un equilibrio entre ambas situaciones, estableciendo en las zonas poco circuladas o frías, actividades que sean muy demandadas y con ello garantizar una diminución del flujo de circulación en las zonas calientes.

Ofertas y promociones: se realizan promociones de acuerdo con las preferencias de los clientes, se cuelgan en las paredes, el mobiliario o el techo del establecimiento. Actualmente, los consumidores otorgan un gran valor a aspectos como la inmediatez entre la compra y el refuerzo, y la comodidad y la sencillez para obtener la promoción (cuánto menos esfuerzo tenga que realizar para conseguir el beneficio, mayor el interés del cliente).

Como se pudo apreciar anteriormente, la publicidad y las TIC juegan un papel determinante en el incremento de las ventas, por lo que, en este sentido, resulta imprescindible garantizar la existencia de promociones atractivas y personalizadas según las características de los locales, el contexto, los servicios o productos, etc. Se debe evitar la utilización de corrientes y patrones acostumbrados, así como los pequeños formatos, la propaganda de productos inexistentes o fuera de época, etc. Otra cuestión de suma importancia, es garantizar la creación de sitios web corporativos y generalizar las ofertas en las redes sociales, denominado comúnmente ventas online.

Animación del punto de venta: conjunto de acciones promocionales que se celebran en un establecimiento durante un tiempo determinado con motivo de un acontecimiento particular, por ejemplo: inicio del curso escolar, día de las madres, día de San Valentín, navidad y fin de año, etc., puede ser desarrollada a través de cuatro medios:

1. Medio físico: utilización de publicidad en sus diferentes formas, como exhibidores, embalajes presentadores y carteles. Publicidad directa mediante la utilización de catálogos y folletos.

2. Medios psicológicos: promociones y colocación repetitiva del producto.

3. Medios de estímulos: audiovisuales que captan la atención del cliente.

4. Medios personales: degustaciones, animadores en el punto de venta (personajes ficticios).

La publicidad es un aspecto diferenciador dirigido a captar la atención de los clientes e incentivar la compra, ello se enriquece aún más cuando empleamos una animación oportuna en el punto de venta, lo que a nivel de ciudad puede verse cuando se realizan carnavales, festivales danzarios o teatrales en las calles, etc., y el público interactúa 
directamente con las mascotas, los artistas y/o participan además en el resto de las actividades programadas. Esto, en el plano propio de los establecimientos comerciales, puede constituir un atractivo no explotado que será necesario implementar de inmediato.

Elección del surtido y presentación de los artículos: el surtido debe adaptarse a las características de la clientela del establecimiento. La elección del mismo, su amplitud (variedad de categorías del producto) y profundidad (variedad de marcas de cada categoría), debe realizarse teniendo en cuenta los estudios que revelan la naturaleza del mercado, conociendo que se va abastecer, las necesidades, la competencia existente y la imagen que se quiere posicionar en el establecimiento.

Esta regla, desde el enfoque de ciudad, se refiere a la necesidad de identificar oportunamente que tipos de productos y servicios serán los más oportunos en un determinado lugar de la urbe, cuáles son los más rentables. Se trata de estudiar las características de consumo de la clientela habitual y determinar qué productos y servicios perdieron su atractivo, así como identificar necesidades aún insatisfechas.

Localización de los productos: habitualmente, la localización de los productos requiere ser variada con frecuencia. Si observamos grandes superficies de ventas, estas suelen cambiar la disposición de los productos cada cierto tiempo. Esto se debe a que los consumidores memorizan el recorrido y van directo a buscar los productos deseados sin pararse a mirar el resto de los que se encuentran en el camino. Esto tiene un impacto negativo en el número de las ventas impulsivas. No obstante, no se debe abusar de este tipo de recursos y siempre que se lleve a cabo, no se puede olvidar modificar también la señalización de los productos.

A nivel de metrópolis, resulta un poco difícil cambiar la ubicación de los locales y estructuras administrativas con frecuencia, mucho más en el caso de los servicios, es por ello que en el proceso de planificación o inversión, debe definirse claramente la opción más adecuada para cada uno de los componentes que conforman la urbe o que pasarán a formar parte de la misma. Cada establecimiento, local, servicio u objeto decorativo, debe ser planificado cuidadosamente sin dejar de considerar ningún aspecto, que pueda afectar su funcionabilidad y en especial, analizar como interactuará con el contexto y la propia armonía urbanística de la ciudad.

\section{Conclusiones}

Se logra cumplir el objetivo de la investigación, disponiéndose de una base teórica elemental sobre la posibilidad de aprovechar el merchandising, las TIC y la publicidad de manera integral en la gestión de las ciudades turísticas, partiendo de los detalles que fidelizan y contribuyen al incremento de la rentabilidad, como vía para el logro de un desarrollo sostenible y perdurable, que asegure el tránsito hacia una ciudad más inteligente y un país más competitivo. 


\section{Referencias Bibliográficas}

BLAYA, Y. ¿Qué es un vlog?. [en línea]. Ensalza-Agencia de Desarrollo y Diseño Web, 2018. [Consulta: 2 de agosto de 2018]. Disponible en: https://blog.ensalza.com/diccionario/que-es-un-vlog/

BOUSKELA, M. [et al.] La ruta hacia las smart cities: Migrando de una gestión tradicional a la ciudad inteligente. Madrid, España: Ed. Banco Interamericano de Desarrollo (BID), 2016.

BREVA, E. y MUT, M. El desarrollo de las ciudades desde la publicidad exterior y las neurociencias. Revista Opción. [en línea] 32 (7): 1-6, 2016. [Consulta: 13 de agosto de 2018]. Disponible en: http://www.redalyc.org/articulo.oa?id=31048480016. ISSN 1012-1587.

CABEZAS, E. Conceptualización para el diseño e introducción de un nuevo producto turístico. Revista Turismo al Día. 10 (3), septiembre 2017. ISSN 2076-2844.

CABEZAS, E. Propuesta de material de apoyo, guía didáctica y programa docente para el curso de: Comercialización del producto turístico. Revista Visión CIDTUR, (9), noviembre 2017. ISSN 1727-1495.

CABEZAS, E. Aprovechamiento de las TICS en el aprendizaje de las reglas y técnicas del merchandising. Revista Visión CIDTUR, (1), julio 2018. ISSN 1727-1495.

CABEZAS, E. Expectativa vs realidad, el impacto de la información gráfica en la comercialización del producto turístico cubano. Revista Turismo al Día, "aceptado para publicación".

CABEZAS, E. El merchandising, las Tics y la publicidad desde una perspectiva de ciudad. Memorias Encuentro Internacional - Gestión de Ciudades Patrimoniales 2018, "aceptado para publicación”.

CABEZAS, E. La información en el merchandising, su valor en la acción de compra. Memorias 2do Taller de Jóvenes Profesionales de la Información de la SOCICTE, "aceptado para publicación".

CABRERA, B. Estrategia pedagógica para el mejoramiento del desempeño profesional pedagógico con el aprovechamiento de las TIC por los docentes de la UCACUE. Tesis (en opción al grado científico de Doctor en Ciencias Pedagógicas), La Habana: Universidad de La Habana, 2017.

CUENCA, M. Ocio humanista. Documentos de ocio, 16. España: Ed. Universidad de Deusto, 2000.

DUPEYRAS, A. y MACCALLUM, N. Indicators for Measuring Competitiveness in Tourism: A Guidance Document [en línea]. Organisation for Economic Co- 
operation and Development, 2013. [Consulta: 4 de agosto de 2018]. Disponible en: http://dx.doi.org/10.1787/5k47t9q2t923-en

GÓMEZ, G. Procedimiento metodológico de diseño de productos turísticos. Revista Retos. 4 (8) 2014.

GONZÁLEZ, S. La importancia del merchandising como estrategia de comunicación y ventas: caso práctico Leroy Merlin. Tesis (de grado en publicidad y relaciones públicas), Valladolid: Universidad de Valladolid, 2015.

INTALENTIA INNOVACIÓN, S. L. U. Merchandising [en línea]. 2013. [Consulta: 17 de abril de 2018]. Disponible en: http://ww.intalentia.com/wpcontent/uploads/2013/07/MERCHANDISING.pdf

LEÓN, F. ¿Qué es el merchandising y cuál es su importancia en marketing? Revista Merca2.0 [en línea]. 2015. [Consulta: 4 de junio de 2018]. Disponible en: http://www.merca20.com

LUKSDESIGNSHOP. (2013). Divide y Vencerás: Productos Estrella, Imán o Impulso. Visual Merchandising [en línea]. Luks, 2015. [Consulta: 10 de agosto de 2018]. Disponible en: http://www.luksandcompany.com/divide-y-venceras-productosestrella-iman-o-impulso/

MARTÍNEZ, Y. Metodología para la evaluación integradora del aprendizaje autónomo de inglés con el aprovechamiento de un entorno personal de aprendizaje en la UCI. Tesis (en opción al grado científico de Doctor en Ciencias de la Educación), La Habana: Universidad de La Habana, 2015.

MEDINA, Y. Cuba y el impacto de las TIC en la informatización de la sociedad [en línea]. Consolación del Sur, Pinar del Rio: Centro Universitario Municipal: Hermanos Saíz Montes de Oca, 2016. [Consulta: 15 de mayo de 2017].

Disponible en: http://www.mercaticaytecnologia.com/trabajos10/PDF/2134943917/cuba-yimpacto-tic-informatizacion-sociedad/

MERCHANDISING PERÚ. Siete técnicas de merchandising para puntos de venta [en línea]. Perú, 2015. [Consulta: 30 de noviembre de 2017]. Disponible en: http://merchandising-p.blogspot.com/2015/09/siete-tecnicas-de-merchandisingpara.html

MORALES, M. D. L. Á. Dossier competencia organización de la tienda. La Habana, Cuba: Ed. FORMATUR, 2014.

MOUTON, D. Merchandising estratégico. Barcelona, España: Ed. Gestión 2000, 1989. 
RIVERO, M. Modelo teórico-metodológico para la formación integral de los estudiantes de primer año de la Universidad de las Ciencias Informáticas mediante la enseñanza de la Matemática Discreta en espacios virtuales. Tesis (en opción al grado científico de Doctor en Ciencias de la Educación), La Habana Universidad de las Ciencias Informáticas, 2011.

ROSENFIELD, K. ¿Qué hace atractiva a una ciudad? Prueba con estos 6 puntos [en línea]. 2015. [Consulta: 23 de agosto de 2018]. Disponible en: https://www.plataformaarquitectura.cl/cl/763950/que-hace-atractiva-a-unaciudad-prueba-con-estos-6-puntos

SALEN, H. Los secretos del merchandising activo o cómo ser el número 1 en el punto de venta. Madrid, España: Ed. ESIC, 1994.

SALVOLOMAS. La belleza de las ciudades es objetiva - Alain de Botton - video [en línea]. 2015. [Consulta: 23 de agosto de 2018]. Disponible en: https://salvolomas.wordpress.com/2015/03/23/la-belleza-de-las-ciudades-esobjetiva-alain-de-botton-video/

TRUJILLO, J. A. La superación profesional del docente mediado por las Tecnologías de la Información y las Comunicaciones, desde una modalidad de la Educación abierta y a Distancia. Tesis (en opción al grado científico de Doctor en Ciencias Pedagógicas), La Habana: Universidad de Ciencias Pedagógicas "Rafael María De Mendive", 2015.

VALCÁRCEL, N. [et al.]. Investigación Educativa (Compilación). La Habana, Cuba: Ed. Universidad de Ciencias Pedagógicas Enrique José Varona, 2015.

\section{¿Ciencia}




\section{Para citar el artículo indexado.}

Cabezas E. (2018). El merchandising, las TIC y la publicidad en la gestión de ciudades turísticas. Revista electrónica Explorador Digital 2(4), 48-63. Recuperado desde: http://cienciadigital.org/revistacienciadigital2/index.php/exploradordigital/article/view/340/7 52

\section{Ciencia Digital \\ Editorial}

El artículo que se publica es de exclusiva responsabilidad de los autores y no necesariamente reflejan el pensamiento de la Revista Explorador Digital.

El articulo queda en propiedad de la revista y, por tanto, su publicación parcial y/o total en otro medio tiene que ser autorizado por el director o editor de la Revista Explorador Digital.
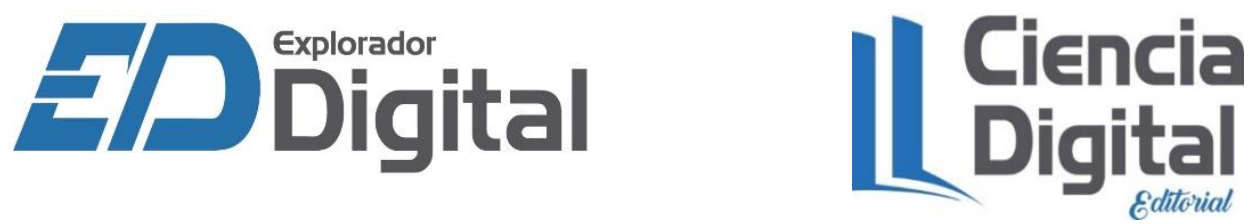